\title{
Factors associated with bruxism in children with developmental disabilities
}

\section{Valeska Aparecida Fernandes de SOUZA(a) \\ Mauro Henrique Nogueira \\ Guimarães ABREU(b) \\ Vera Lúcia Silva RESENDE(a) \\ Lia Silva de CASTILHO(a)}

(a) Universidade Federal de Minas Gerais UFMG, School of Dentistry, Department of Operative Dentistry, Belo Horizonte, MG, Brazil.

(b) Universidade Federal de Minas Gerais UFMG, School of Dentistry, Department of Community and Preventive Dentistry, Belo Horizonte, MG, Brazil.
Declaration of Interests: The authors certify that they have no commercial or associative interest that represents a conflict of interest in connection with the manuscript.

\section{Corresponding Author:}

Mauro Henrique Nogueira Guimarães Abreu E-mail: maurohenriqueabreu@ig.com.br

DOI: 10.1590/1807-3107BOR-2015.vol29.0009

Submitted: Apr 24, 2014

Accepted for publication: Aug 27, 2014

Last revision: Oct 16, 2014
Abstract: The aim of the present study was to investigate factors associated with bruxism in children aged from 1 to 13 years with developmental disabilities. A total of 389 dental records were examined. The bruxism analyzed was determined based on parental reports. The following variables were also analyzed: gender, age, International Code of Diseases (ICD), mouth breathing, history of gastroesophageal reflux, use of psychotropic drugs, gingival status, reports of xerostomia, hyperkinesis, pacifier use, thumb sucking and involuntary movements. For the purposes of analysis, the individuals were categorized as being with and without bruxism. Variables with a $p$-value $<0.25$ in the bivariate analysis were incorporated into the logistic regression models. Females had a 0.44 -fold $(95 \% \mathrm{CI}: 0.25$ to $0.78)$ greater chance of exhibiting bruxism than males. Individuals with gastroesophageal reflux had a 2.28 -fold $(95 \% \mathrm{CI}$ : 1.03 to 5.02$)$ greater chance of exhibiting bruxism. Individuals with reported involuntary movements had a 2.24-fold (95\%CI: 1.19 to 4.24) greater chance of exhibiting bruxism than those without such movements. Exhibiting involuntary movements, the male gender and gastroesophageal reflux are factors associated with bruxism in children with developmental disabilities.

Keywords: Dental Care for Disabled; Developmental Disabilities; Bruxism; Cerebral Palsy.

\section{Introduction}

Bruxism is the involuntary act of clenching or grinding one's teeth, either while awake or asleep, in an occasional to constant manner. Sleep bruxism is more common than awake bruxism. ${ }^{1}$ According to a systematic review, the prevalence ranges from $5 \%$ to $40 \%$ around the world. This discrepancy in prevalence is most likely due to the different measuring instruments used in bruxism diagnosis. ${ }^{2}$ In Brazil, the prevalence is $35.3 \%$ in the general pediatric population. ${ }^{3}$ In children and adolescents with mental health problems (emotional symptoms, behavioral problems, peer problems, hyperactivity, etc.), the rate ranges from $28.7 \%$ to $30.0 \%{ }^{4}$ Among children with developmental disabilities, such as cerebral palsy, the prevalence ranges from $25.0 \%$ to $69.4 \% .5,6,7,8$ In studies involving children with cerebral palsy and a control group, a higher prevalence rate has been reported in the former group, whereas other studies have found no significant differences between groups. ${ }^{6}$

In individuals with cerebral palsy, bruxism has been associated with spastic quadriplegia, athetosis, ${ }^{5}$ sucking habits, posterior crossbite, ${ }^{6}$ gastroesophageal reflux ${ }^{10}$ and Level III of the Gross Motor Functional 
Classification System. ${ }^{11}$ Together with dental caries, bruxism has a negative impact on the quality of life of this group of individuals. ${ }^{8}$

Knowledge regarding factors associated with bruxism among individuals with developmental disabilities can assist in broadening the understanding of this condition and the establishment of treatment options that are more suitable for this group of patients. Thus, the purpose of the present study was to investigate factors associated with bruxism in children aged 1 to 13 years with developmental disabilities treated at a reference service health care service for patients with special needs in the city of Belo Horizonte, Brazil.

\section{Methodology}

This study received approval from the Human Research Ethics Committee of of the Universidade Federal de Minas Gerais - UFMG (process no: ETIC 219/03). A cross-sectional, epidemiological study was carried out using information collected from dental charts obtained from a reference center for the rehabilitation of children with neuromotor disabilities, which is affiliated with the aforementioned university and the Associação Mineira de Reabilitação - AMR.

A total of 389 charts were examined of children aged from 1 to 13 years with developmental disabilities (cerebral palsy, intellectual disability) who were treated between January 1998 and December 2013. The sample size was calculated based on $95 \%$ confidence interval, $80 \%$ power, and frequency of bruxism between each of the covariates from the pilot study with 40 dental charts. This pilot study also allowed for the testing of the instruments.

The frequency of thumb sucking was used in the sample size calculation because it resulted in the highest number of individuals. Data on the outcome and independent variables were simultaneously extracted from the patient charts by the same trained observer without any need for reexamination. Although this study does not provide an exact measure of intra-rater agreement, the observer consistently followed the daily routine employed in this center. Examiners attended at least two hours of lecture and two hours of practical demonstrations before starting dental care. For analysis purposes, the individuals were categorized as being with or without bruxism experience based on reports by parents/caregivers (Does your child have audible teeth grinding?). Sleep bruxism was not differentiated from awake bruxism. The parental report of bruxism was used for the calculation of the prevalence rate, as performed in previous studies. ${ }^{4,6,12,13,14,15,16,17}$ The following variables were also analyzed: gender, age, International Code of Diseases (ICD), history of gastroesophageal reflux, use of psychotropic drugs, gingival status, reports of xerostomia, hyperkinesis, pacifier use, thumb sucking and involuntary movements. The ICD of the neurological condition of each patient was defined by the physician in charge. The different diagnoses of spasticity were pooled into a single category. Other developmental disabilities were grouped into another category. The remaining variables were dichotomized as being either absent or present. With the exception of the medical diagnosis, which was defined during neurological treatment, the other variables were measured on the first day of dental treatment and a clinical chart that was created to serve as a basis for the dental treatment proposed for each patient.

The data were submitted to simple logistic regression for the determination of associations, with the calculation of respective odds ratios (ORs) and $p$-values. Variables with a $p$-value $<0.25$ in this analysis were incorporated into the multiple logistic regression models. Variables with a $p$-value $<0.05$ were maintained in the final model. Adjusted ORs with the respective $95 \%$ confidence intervals (CIs) and $p$-values were calculated. All statistical analyses were performed using SPSS version 19.0 (SPSS Inc., Chicago, USA).

\section{Results}

A total of 389 charts of children aged from 1 to 13 years were analyzed. Among those, the information on bruxism was available in 369 (Response rate $=94.9 \%$ ). Bruxism was reported by parents/caregivers in 36.3\% of the cases. The simple logistic regression revealed that the following variables were associated with bruxism $(p<0.05)$ : neurological diagnosis, gender, report of gastroesophageal reflux, mouth breathing and involuntary movements $(p<0.05)$. The multiple logistic regression model revealed that females had a 0.44 -fold ( $95 \% \mathrm{CI}: 0.25$ to 0.78$)$ greater chance of exhibiting bruxism than males. Individuals with gastroesophageal reflux had a 2.28 -fold (95\%CI: 1.03 to 5.02 ) greater chance of exhibiting 
bruxism. Individuals with reported involuntary movements had a 2.24-fold (95\%CI: 1.19 to 4.24 ) greater chance of exhibiting bruxism than those without such movements (Table 1). The Hosmer-Lemersshow test demonstrated that the logistic regression model was adequate $(p=0.749)$.

\section{Discussion}

Gender, gastroesophageal reflux and involuntary movements were independently associated with parent/caregiver-reported bruxism in the children analyzed in the present study.

While a number of previous studies have reported no association between bruxism and gender, ${ }^{13,14,15,18,19}$ one study found such an association and reported that the strength of the association with the male gender diminished with age. ${ }^{12} \mathrm{~A}$ similar finding was reported in another study in which the authors suggest that girls tend to be less aggressive and agitated than boys and, due to social impositions, boys are unable to express their feelings, whereas girls express their feelings mainly through crying. ${ }^{4}$ All studies cited involved questionnaires administered to parents/caregivers. With respect to self-perceptions regarding bruxism, female adolescents tended to report the problem more often. ${ }^{1}$

The association between gastroesophageal reflux and bruxism was in agreement with findings reported in previous studies ${ }^{10,20}$ but differed from data described for individuals with cerebral palsy. ${ }^{7}$ An increase in the rhythmic activity of the masticatory muscles, salivary flow and swallowing has been demonstrated through experimental acidification in healthy adults. Based on these data, bruxism (especially sleep bruxism) may follow gastroesophageal reflux, with a consequent increase in the excitation of the masticatory muscles and swallowing reflex..$^{10}$ Sleep bruxism also leads to excitation during sleep and increased swallowing. The aim of this increase in salivation is to neutralize the

Table 1. Associations between reported bruxism and independent variables

\begin{tabular}{|c|c|c|c|c|c|c|}
\hline Variable* & Without bruxism & With bruxism & Crude OR $(95 \% \mathrm{Cl})$ & $p$-value & Adjusted OR (95\%Cl) & $p$-value \\
\hline \multicolumn{7}{|c|}{ Diagnosis of spasticity } \\
\hline No & $34(79.1 \%)$ & 9 (20.9\%) & 1 & 0.020 & & \\
\hline Yes & $136(59.9 \%)$ & 91 (40.1\%) & $2.53(1.16-5.52)$ & & & \\
\hline \multicolumn{7}{|l|}{ Gender } \\
\hline Male & $116(56.9 \%)$ & $88(43.1 \%)$ & 1 & 0.003 & 1 & 0.005 \\
\hline Female & $119(72.1 \%)$ & $46(27.9 \%)$ & $0.51(0.33-0.79)$ & & $0.44(0.25-0.78)$ & \\
\hline \multicolumn{7}{|c|}{ Gastroesophageal reflux } \\
\hline No & $212(66.2 \%)$ & 108 (33.8\%) & 1 & 0.015 & 1 & 0.041 \\
\hline Yes & $23(47.9 \%)$ & $25(52.1 \%)$ & $2.13(1.16-3.93)$ & & $2.28(1.03-5.02)$ & \\
\hline \multicolumn{7}{|c|}{ Mouth breathing } \\
\hline No & $128(69.6 \%)$ & $56(30.4 \%)$ & 1 & 0.025 & & \\
\hline Yes & $106(58.2 \%)$ & $76(41.8 \%)$ & $1.64(1.06-2.52)$ & & & \\
\hline \multicolumn{7}{|c|}{ Psychotropic drugs } \\
\hline No & $128(67.7 \%)$ & 61 (32.3\%) & 1 & 0.081 & & \\
\hline Yes & $100(58.8 \%)$ & $70(41.2 \%)$ & $1.47(0.95-2.27)$ & & & \\
\hline \multicolumn{7}{|c|}{ Pacifier sucking } \\
\hline No & 135 (61.4\%) & 85 (38.6\%) & 1 & 0.212 & & \\
\hline Yes & $62(68.9 \%)$ & $28(31.1 \%)$ & $0.71(0.42-1.21)$ & & & \\
\hline \multicolumn{7}{|c|}{ Thumb sucking } \\
\hline No & $170(64.9 \%)$ & $92(35.1 \%)$ & 1 & 0.094 & & \\
\hline Yes & $25(52.1 \%)$ & $23(47.9 \%)$ & 1.70 (0.91-3.17) & & & \\
\hline \multicolumn{7}{|c|}{ Involuntary movements } \\
\hline No & $151(66.8 \%)$ & 75 (33.2\%) & 1 & 0.009 & 1 & 0.013 \\
\hline Yes & $39(50.0 \%)$ & $39(50.0 \%)$ & $2.01(1.19-3.40)$ & & $2.24(1.19-4.25)$ & \\
\hline
\end{tabular}

*Some variables have missing data. 
lower $\mathrm{pH}$ of the esophagus due to acidification from the stomach in cases of gastroesophageal reflux..$^{20}$

The association between bruxism and involuntary movements may be explained by mechanisms of the central nervous system. Neurological impairment is common among individuals with developmental disabilities and can trigger biting behavior as a pattern of primitive oral reflexes. ${ }^{9}$ This mechanism seems to involve direct and indirect pathways of the basal ganglia, which consist of a group of five subcortical nuclei involved in the coordination of movements and are altered in individuals with bruxism. ${ }^{21}$ Despite the plausibility of this explanation, further studies are needed for a more complete understanding of this association.

Other factors measured in the present study, such as thumb and pacifier sucking, hyperactivity and the use of psychotropic drugs were not associated with the outcome. These findings are in disagreement with data reported in previous studies. ${ }^{4,6,15,22}$ No association with age was found, which is in agreement with findings described in previous studies. ${ }^{1,3,6}$ Moreover, no association with mouth breathing was observed, although previous studies have found such an association. ${ }^{16,23}$

Some variables reported to be associated with bruxism in the literature were not addressed in the present study, such as stress, level of responsibility, 3,15,17,19 excessive salivation, sleep walking, heredity, ${ }^{13,15}$ the habit of chewing gum, biting on objects, ${ }_{19}^{19}$ headache, ${ }^{24}$ occlusal factors, impacted food scraps and extensive tooth decay. ${ }^{13}$

The frequency of bruxism was similar to that reported in previous studies, ${ }^{3,4,9,14}$ higher than rates found in $\operatorname{Iran}^{13,15}$ and lower than rates found in studies involving individuals with cerebral palsy. ${ }^{5,8,11}$ The divergence between the rate in the present investigation and those reported in studies involving individuals with cerebral palsy may be due to the small sample sizes in the studies cited. A systematic review of the literature reports that the prevalence rate of bruxism ranges from $5 \%$ to $40 \%$, with higher rates found in Brazil in comparison to rates reported throughout the world. ${ }^{2}$ The $37.9 \%$ rate in the present study may have been influenced by the specific characteristics of the sample (individuals with developmental disabilities treated at a health care service).

Basing on the prevalence of bruxism on parental reports can be considered to be a limitation of the present study. In a previous study, the prevalence rate was $69.4 \%$ when based on a clinical exam and $57.0 \%$ when based on the reports of parents/caregivers. ${ }^{5}$ However, the methodology employed herein has been previously used for groups with cerebral palsy as well as for the general population. ${ }^{3,4,6,8,9,11,12,13,14,15,16,17,24}$ Indeed, a diagnosis based on tooth wear should be interpreted with caution, as individuals who have recently acquired the habit are not likely to exhibit typical signs of tooth wear. The cross-sectional design is another limitation, as this type of study does not allow for the determination of cause and effect. Thus, prospective analytical studies are needed to gain a better understanding of this phenomenon. The nondifferentiation of sleep bruxism from awake bruxism is another limitation. However, many of these children clearly grind their teeth throughout the entire day and/or night. Indeed, a previous study found no significant differences in the comparison of groups with sleep bruxism, those with awake bruxism and those who exhibited both behaviors, and it found no increased risk for the temporomandibular joint, sleep disorders or behavioral complaints when sleep and awake bruxism coexisted. ${ }^{24}$

Identifying factors that may predict the occurrence of bruxism in children with developmental disorders are very important to identify groups with a greater chance of having this condition. The treatment of bruxism involves behavioral aspects, such as anxiety control and improving sleep. Psychotherapy, orthodontics, speech therapy, physical therapy and drug therapy can also assist in the treatment of this condition..$^{5,17,18}$ The construction of bite plates is difficult due to the complex molding stages and the establishment of centric occlusion in these patients. Moreover, the use of the plate itself poses problems, as the growth of the dental arches is not yet complete in children and the plate may break or even be swallowed. Understanding the factors associated with this condition in a group that poses particular challenges to dental care is important to establish the best treatment approach. 


\section{Conclusions}

Exhibiting involuntary movements, male gender and gastroesophageal reflux are factors associated with bruxism in children with developmental disabilities.

\section{References}

1. Van Selms MKA, Visscher CM, Naeije M, Lobbezoo F. Bruxism and associated factors among dutch adolescents. Community Dent Oral Epidemiol. 2013 Aug;41(4):353-63.

2. Manfredini D, Restrepo C, Diaz-Serrano K, Winocur E, Lobbezoo F. Prevalence of sleep bruxism in children: a systematic review of the literature. J Oral Rehabil. 2013 Aug;40(8):631-42.

3. Serra-Negra JM, Paiva SM, Abreu MH, Flores-Mendonza CE, Pordeus IA. Relationship between tasks performed, personality traits, and sleep bruxism in Brazilian school children - a population-based cross-sectional study. PLoS One. 2013 Nov 14;8(11):1-6. e80075. DOI: 10.1371/journal.pone.0080075. eCollection 2013.

4. Renner AC, Silva AAM, Rodriguez JDM, Simões VMF, Barbieri MA, Bettiol H, et al. Are mental health problems and depression associated with bruxism in children?. Community Dent Oral Epidemiol. 2012 Jun;40(3):277-87.

5. Peres ACD, Ribeiro MO, Juliano Y, Cesar MF, Santos RCA. Occurrence of bruxism in a sample of Brazilian children with cerebral palsy. Spec Care Dentist. 2007 Mar-Apr;27(2):73-6.

6. Miamoto CB, Pereira LJ, Ramos-Jorge ML, Marques LS. Prevalence and predictive factors of sleep bruxism in children with and without cognitive impairment. Braz Oral Res. 2011 Sep-Oct;25(5):439-45.

7. Guare RO, Ferreira MCD, Leite MF, Rodrigues JA, Lussi A, Santos MTBR. Dental erosion and salivary flow rate in cerebral palsy individuals with gastroesophageal reflux. J Oral Pathol Med. 2012 May;41(5):367-71.

8. Abanto J, Ortega AO, Raggio DP, Bönecker M, Mendes FM, Ciamponi AL. Impact of oral diseases and disorders on oralhealth-related quality of life of children with cerebral palsy. Spec Care Dentist. 2014 Mar;34(2):56-63.

9. Ortega AOL, Guimarães AS, Ciamponi AL, Marie SKN. Frequency of parafunctional oral habits in patients with cerebral palsy. J Oral Rehabil. 2007 May;34(5):323-8.

10. Mengatto CM, Dalberto CS, Shereen B, Barros SGS. Association between sleep bruxism and gastroesophageal reflux disease. J Prosthet Dent. 2013 Nov;110(5):349-55.

11. Elsayed RM, Hasanein BM, Sayyah HE, El-Auoty MM, Tharwat N, Belal TM. Sleep assessment of children with cerebral palsy: Using validated sleep questionnaire. Ann Indian Acad Neurol. 2013 Jan;16(1):62-5.

12. Lam MHB, Zhang J, Li AM, Wing YK. A community study of sleep bruxism in Hong Kong children: association with comorbid sleep disorders and neurobehavioral consequences. Sleep Med. 2011 Aug;12(7):641-5.

\section{Acknowledgement}

This article was supported by Pró-Reitoria de Pesquisa of Universidade Federal de Minas Gerais - UFMG.
13. Ghafournia M, Hajenourozali Tehrani M. Relationship between bruxism and malocclusion among preschool children in Isfahan. J Dent Res Dent Clin Dent Prospects. 2012 Fall;6(4):138-42.

14. Cheifetz AT, Osganian SK, Allred EN, Needleman HL. Prevalence of bruxism and associated correlates in children as reported by parents. J Dent Child. 2005 May-Aug;72(2):67-73.

15. Seraj B, Shahrabi M, Ghadimi S, Ahmadi R, Nikfarjam J, Zayeri $\mathrm{F}$, et al. The prevalence of bruxism and correlated factors in children referred to dental schools of Tehran, based on parents' report. Iran J Pediatr. 2010 Jun;20(2):174-80.

16. Grechi TH, Trawitzki LVV, Felicio CM, Valera FCP, AnselmoLima WT. Bruxism in children with nasal obstruction. Int J Pediatr Otorhinolaryngol. 2008 Mar;72(3):391-6.

17. Serra-Negra JM, Paiva SM, Flores-Mendonza CE, RamosJorge ML, Pordeus IA. Association among stress, personality traits, and sleep bruxism in children. Pediatr Dent. 2012 Mar-Apr;34(2):e30-4.

18. Ghanizadeh A, Zare S. A preliminary randomised double-blind placebo-controlled clinical trial of hydroxyzine for treating sleep bruxism in children. J Oral Rehabil. 2013 Jun;40(6):413-7.

19. Cortese SG, Fridman DE, Farah CL, Bielsa F, Grinberg J, Biondi AM. Frequency of oral habits, dysfunctions, and personality traits in bruxing and nonbruxing children: a comparative study. Cranio. 2013 Oct;31(4):283-90.

20. Miyawaki S, Tanimoto Y, Araki Y, Katayama A, Fujii A, Takano-Yamamoto T. Association between nocturnal bruxism and gastroesophageal reflux. Sleep. 2003 Nov 1;26(7):888-92.

21. Shetty S, Pitti V, Babu CLS, Kumar GPS, Deepthi BC. Bruxism: a literature review. J Indian Prosthodont Soc. 2010 Sep;10(3):141-8.

22. Lavigne GJ. Kato T, Kolta A, Sessle BJ. Neurobiological mechanisms involved in sleep bruxism. Crit Rev Oral Biol Med. 2003 Jan;14(1):30-46.

23. Eftekharian A, Raad N, Gholami-Ghasri N. Bruxism and adenotonsillectomy. Int J Pediatr Otorhinolaryngol. 2008 Apr;72(4):509-11.

24. Carra MC, Huynh N, Morton P, Rompre PH, Papadakis A, Remise $\mathrm{C}$, et al. Prevalence and risk factors of sleep bruxism and wake-time tooth clenching in a 7- to 17-yr- old population. Eur J Oral Sci. 2011 Oct;119(5):386-94. 